\title{
AN EFFICIENT METHOD TO AUTOMATE POTHOLE DETECTION IN ROAD SURFACE USING IOT
}

\author{
Mr. Ajith Kumar B, Mr. Vignesh .G (UG Students) \\ Department of Computer Science and Engineering \\ Velammal Institute of Technology \\ Panchetti, Tamil Nadu.
}

\begin{abstract}
With the development of information technology, the digital image processing has the characteristics of strong permeability, large use of action and good comprehensive benefits. A road maintenance pothole detection is one of the important tasks. A road surface modelling or road image analysis is generally come from computer vision approaches. However, these two categories were always used independently. Furthermore, the accuracy of the pothole detection is not satisfactory. These challenges promote the development of a better application to detect potholes, cracks using the digital image processing like segmentation, extraction, recognition, and morphology from the images of road surface by using image processing. We are proposing an application system with efficient digital image processing techniques to improve the accuracy and consistency of obtaining accurate shapes of potholes and topologies, etc. The successful detection accuracy is around $98.7 \%$ and the overall pixel-level accuracy is approximately $99.6 \%$. By using the digital image processing techniques, the detected potholes and cracks are updated to the web server by using IOT device.
\end{abstract}

Keywords - Crack detection, Disparity map, OTSU Threshold, Morphology, Web server update.

\section{INTRODUCTION}

Road potholes are the cause of large structural failures on the road surface. Contraction and expansion of the road surface can cause rain water to permeate into the ground. Road potholes must be frequently inspected and repaired in which traffic safety can be ensured. Currently, certified inspectors and structural engineers use manual methods to find and track potholes regularly. The detection results are always subjective, because they purely depend upon their personal experience. As a result, automated pothole detection systems have been developed to efficiently and objectively to identify and locate potholes. Various technologies like active and passive sensing are used

\author{
Mr. Anbumani. A, M.Tech (Assistant Professor) \\ Department of Computer Science and Engineering \\ Velammal Institute of Technology \\ Panchetti, Tamil Nadu.
}

before and that have been utilized to acquire road data and aid personal in detecting road potholes. For example, Tsai and Chatterjee used two laser scanner that were mounted on a digital inspection vehicle (DIV) to collect 3D road surface data. Using, semi or fully automatic methods for pothole detection these data were processed. Such systems ensure personal safety, but manual intervention also needed to be reduced. By comparing the road data collected over different periods, evaluation of traffic flow can be done and future road condition can be predicted. The remainder of this section presents the algorithms of pothole detection, contributions, motivation highlights and the outline of this section.

In recent years, the development of automobile industries has resulted in the mass deployment of automobiles on roads in both developing and developed countries. Although the quality of automobiles now a days has been improved enormously, there are still low-cost models that operate on roads of developing countries. The reason is because expensive models attracts top earners in a region, but the mid-range citizens can only afford low-cost models that operate on roads of developing countries. In order to support automobiles in reducing accidents by hitting potholes on roads, this research aims to developed an improved real-time watershed-based pothole detection algorithm.

The algorithm allows for fully automatic identification of different pothole shapes and sizes on a variety of road surfaces, including smooth, aged, and deteriorated surfaces. Researches have been conducted in, on road object detection using vision sensors. Most common obstacles in road scenarios are either vehicles or pedestrians. Pedestrian detection is lavishly studied in literature and some are reviewed. Different methods used in vehicle detection using vision images are reviewed. All these object categories belong to the super class of 'Positive Obstacles,' which is defined as the class of objects that lies on top of the ground surface. While positive obstacles are the most common, there is another type of obstacles that includes 
holes, ditches, potholes etc. These should also be avoided by an autonomous vehicle. These types of obstacles are summarized as 'Negative Obstacles,' which lie below the ground plane. Autonomous guided vehicles are expected to enter the road in the future and autonomous robots are expected to be deployed to clean roads and pedestrian ways. In fact, there are autonomous robots used in cleaning corridors and path ways. Autonomous robot or vehicle being unable to avoid a negative obstacle can lead to damage itself and surroundings, thus making it a critical component for safety purposes. Furthermore, unattended and open man-hole type negative obstacles are present even when the naturally occurred potholes are absent. On the other hand, absence of natural potholes means there is good maintenance, which requires some mechanism to detect such negative obstacles and take corrective measures our concern is to detect such negative obstacles from binocular stereo images. Literature on pothole detection is not extensive as that of human or vehicle detection. The reasons could be due to the lack of data, especially because of the well-maintained road network in many developed countries and on the other hand unavailability of public datasets with labelled ground truth data for the research community to work on.

\section{LITERATURE REVIEW}

The literature review based on crack detection methodology and GPS location detection

A. This is the paper proposed by Haifeng $\mathrm{Li}$, Dezhen Song, Yu Liu, and Binbin Li in June 2019.

The image pavement crack detection can be difficult due to intensity in-homogeneity, topology complexity, low contrast, and a noisy texture context. Conventional research-based methods fail to achieve representative teaching samples of instruction. A new multi-scale was proposed, unsupervised multi fusion crack detection (MFCD) algorithm that requires no trained data. Candidate cracks were extracted within each scale of the image we first create a windowed minimal intensity path-based process. Second, we look for crack correspondences on various scales. Finally, crack evaluation model is build that can be used in combination with a multi-variate statistical hypothesis test. Our method effectively combines the strengths of both large-scale detection (which is stable and has low localization) and small scale detection (detail conserving but clutter sensitive). The computational complexity of our MFCD algorithm is examined and experimentally tested. The algorithm that we implement and thoroughly tested it on two public pavement data sets, including three public data sets and a runway data set. Experimental results show that our method outperforms all counterparts, compared to six existing methods. Specifically, it increases the precision, recall, and F1 measure over the state of the art by $22 \%, 12 \%$, and $19 \%$, respectively, on one public data set.

\section{B. This is the paper proposed by Andrew Fox,} B.V.K. Vijaya Kumar in December 2017.

Smart vehicle's embedded sensor data now helps them to identify environmental road characteristics as they have become more popular (e.g. road tilt angles, potholes, etc.). Crowd-sourcing can be used to detect environmental information with greater precision by combining data from several vehicles. We focus on finding and locating potholes on multi-lane highways using these data. Information is extracted from aggregated vehicle data is difficult due to under-sampling sensors, sensor-sensitivity, asynchronous sensor operation, sensor noise, vehicle and road heterogeneity, and a GPS position error. In multi-lane environments, error in GPS position is particularly problematic since the location error is typically greater than the usual lane widths. In this paper, we investigate these issues and develop a crowd sourced framework that detects and locates potholes in multi-lane environments using accelerometer data from embedded vehicle sensors. Network bandwidth is reduced from the crowd sourced program needed by calculating inclined road and bank angle information in each vehicle to filter components of the acceleration that do not conform to pothole conditions. Simulated systems and real world data were tested, evaluate tradeoffs in the number of vehicles and the amount of bandwidth required for accurate detection, and results were compared with the simpler single lane detection scenario.

\section{This is the paper proposed by Zhang Yiyang in March 2015.}

This paper describes a glass crack detection algorithm based on digital image processing technology that uses preprocessing, image segmentation, extraction function on the glass crack image, measurement of the target region, and perimeter of the roundness index to determine if the image has a crack. The function is achieved to impolder the crack detection system and the crack detection process, and to use the programming language Visual Basic 6.0.

\section{This is the paper proposed by Dong-Won Jang, Rae-Hong Park in November 2016.}

This research proposes a video-based approach for detecting potholes using spatio temporal saliency, where a pothole is a type of fault in asphalt pavement. In monocular vision, potholes typically have two visual properties. Second, the potholes have low visibility areas that are deeper than the surrounding pavement due to shadows. Second, the texture within the potholes is lower than the nearby pavement. However, due to the lack of texture and shadows on the surface, these two visual properties are insufficient to detect potholes accurately. The above issues 


\section{International Journal of Engineering Applied Sciences and Technology, 2021}

Vol. 5, Issue 10, ISSN No. 2455-2143, Pages 161-170

Published Online February 2021 in IJEAST (http://www.ijeast.com)

are discussed in this research by detecting slower objects that approach a moving vehicle.

\section{E. This is the paper proposed by Yifan Pan, Xianfeng Zhang, Guido Cervone in October 2018.}

Asphalt roads of land transformation are of most basic forms, and their quality can deteriorate over time due to ageing and degradation of the road surface. Finally, road pavement distresses such as potholes and cracks can appear on the road surface. In order to increase pavement inspection performance, no destructive effect on modern types of remote sensing on the pavement are widely used to detect pavement distress, such as digital images, light detection and range, and radar.

\section{PROPOSED WORK POTHOLE DETECTION ALGORITHM}

Disparity map refers to the apparent pixel difference or motion pair between the stereo images. The measure of the apparent motion in pixels for each point in a pair of images derived from stereo cameras, and create an intensity picture out of the measurements. The block diagram of the proposed pothole detection algorithm is illustrated in Fig. 1, where the algorithm consists of two main components: a) Transformation of disparity; b) Extraction of Undamaged Road Surface.

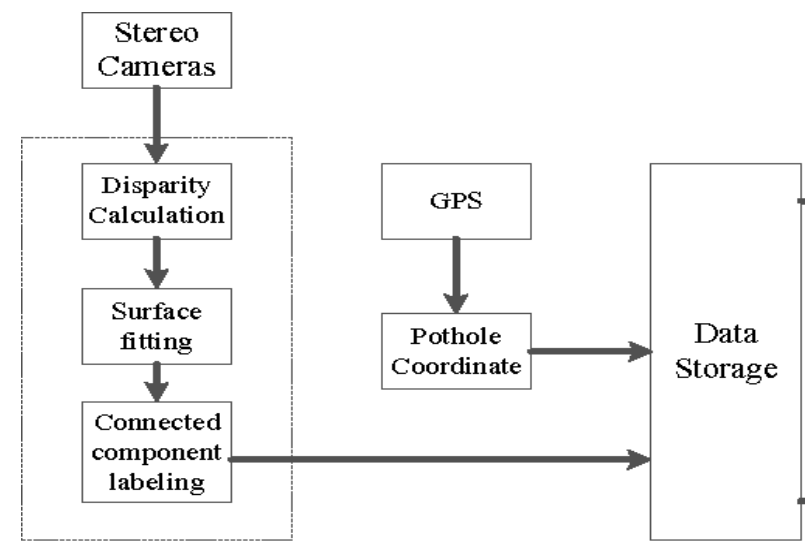

Fig. 1. The block diagram of the proposed pothole detection algorithm.

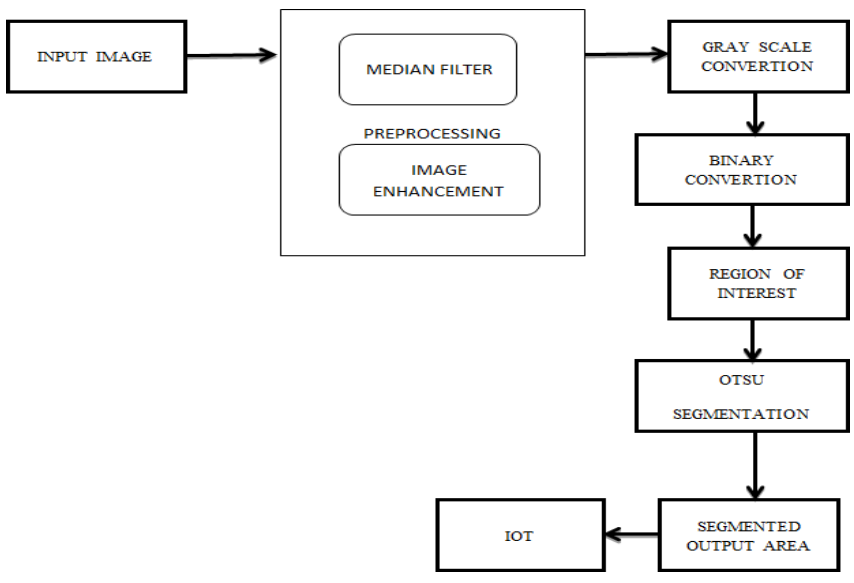

Fig. 2. The Inner Architecture diagram of the proposed pothole detection algorithm

\section{A. Transformation of Disparity}

The input of dense disparity map is accuracy of sub pixel. Since the efficiency of the disparity map modelling is entirely based on the accuracy of the disparity estimation, the dense disparity map was obtained from a stereo road image pair (Fig.3(a)) via our disparity estimation algorithm [12], where the stereo matching search range propagates iteratively from the bottom of the image to the top, and the sub-pixel disparity map is refined via iteration. The map of variance is shown in Fig. 2(b), and displays the related vdisparity map in Fig. 2(c). A v-disparity map can be generated by measuring each horizontal row of the disparity map with a histogram.

The proposed pothole detection algorithm is based on the work described, which models the inequalities of the undamaged road surface using a quadratic surface as follows:

$$
g(u, v)=c_{0}+c_{1} u+c_{2} v+c_{3} u^{2}+c_{4} v^{2}+c_{5} u v,(1)
$$

Where $\mathrm{u}$ and $\mathrm{v}$ are the map coordinates for horizontal and vertical differences, respectively. The root of the scheme of coordinates in (1) is at the middle of the disparity map. The stereo rig experiments are placed at a relatively low height, and the curvature of the reconstructed road surface is not too high. When the stereo rig is completely parallel to the horizontal road surface, this makes the values of $\mathrm{c} 1, \mathrm{c} 3$ and c5 in (1) very close to zero. In this case, it can be concluded that the projection of the road inequalities on the $\mathrm{v}$-disparity map is a parabola of the form:

$$
g(v)=\alpha_{0}+\alpha_{1} v+\alpha_{2} v^{2}
$$

In practice, however, the reference of stereo rigs is not always completely parallel to the horizontal road surface. This reality that introduce in the imaging process a nonzero roll angle $\theta$ where Tc and $\mathrm{h}$ reflect the baseline and the 


\section{International Journal of Engineering Applied Sciences and Technology, 2021 \\ Vol. 5, Issue 10, ISSN No. 2455-2143, Pages 161-170 \\ Published Online February 2021 in IJEAST (http://www.ijeast.com)}

height of the stereo rig, respectively. $o_{l}{ }^{C}$ and $o_{r}{ }^{C}$ are the sources of the coordinated systems for the left and right camera, respectively.

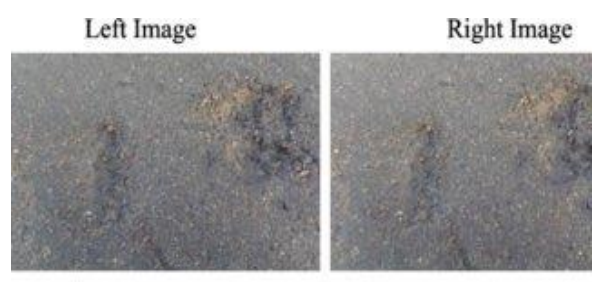

(a)

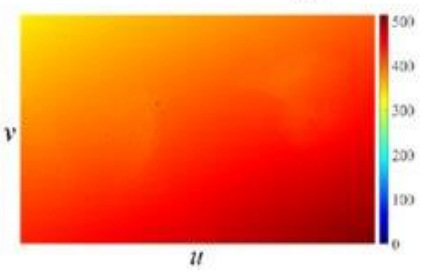

(b)

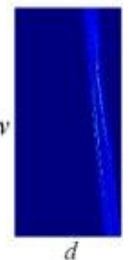

(c)
Fig. 3. Disparity map when the roll angle does not equal zero: (a) stereo road images, (b) disparity map, and (c) v-disparity map.

$O^{W}$ is the coordinate system. An example of the resulting map of inequalities is shown in Fig. 2(b) where readers can see clearly that disparity values gradually change in horizontal direction, rendering the method of representing the projection of disparity quite problematic using (2). In this respect we measure the roll angle value for the first time. The effects induced by the non-zero angle of the roll are then removed by rotating the map of inequalities by means of an alternative. Finally, the disparity projection model coefficients are calculated in (2), and the disparity map is transformed to make a better distinction between damaged and undamaged road areas.

\section{B. Extraction of Undamaged Road Surface}

Next, we use the Otsu thresholding method to segment the transformed disparity map. The segmentation threshold to be obtained by maximizing as follows the inter-class variance $\sigma 02$.

$$
\sigma_{0}{ }^{2}\left(T_{0}\right)=P_{0}\left(T_{0}\right) P_{1}\left(T_{0}\right)\left[\mu_{0}\left(T_{0}\right)-\mu_{1}\left(T_{0}\right)\right]^{2},
$$

where

$$
P_{0}\left(T_{0}\right)=\underbrace{1}_{\substack{d_{i=\text { min }} \\ T_{0}}} p(i), P_{1}\left(T_{0}\right)=\stackrel{d}{\substack{i=\\ T_{0}}}
$$

These depict, respectively, the probabilities of damaged and undamaged road areas. P(i) is the average probability disparity values given for damaged and undamaged road areas by:

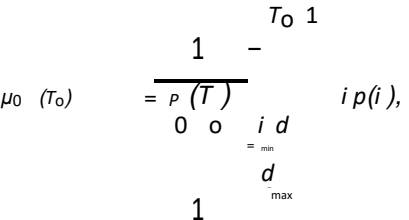

$$
\begin{aligned}
& \mu_{1}\left(T_{0}\right)=\frac{1}{P_{1}\left(T_{0}\right)} \quad i p(i) .
\end{aligned}
$$

We can see that it effectively extracts the undamaged road area. Even though the transformed disparity map contains no road damage, Otsu's thresholding method will still group the disparities into two classes. Then we model the disparity map to ensure that the potholes are identified correctly.

\section{APPROACHES}

The Approaches of the Crack Detection includes two main strategies are a) Crack Detection Techniques; b) Data included in Web Server

\section{A. Crack Detection Techniques}

The Crack Detection techniques involves the following things like image pre-processing, edge detection, OTSU thresholding, morphology.

\section{IMAGE PRE-PROCESSING}

Image pre-processing performs the image operations at the lowest abstraction level both input and output with intensity. These images are iconic with sensors original data which is of same form, with an intensity of image defined by an image function values matrix (brightness). Geometric image transformations (e.g. rotation, scaling, translation) are classified as pre-processing methods here since similar techniques are used. Pre-processing is aimed at improving image data, removing unwanted defects, or other image features enhanced that are required for further processing. Pre-processing is the first stage of any image processing application, and it eliminates unwanted objects from the image or frame captured. Input image will undergo below processes such as
1. Resized
2. Gray scale Conversion

Median Filter is used as a one of image pre-processing technique.

\subsection{MEDIAN FILTER}

The Median Filter is a non-linear digital filtering technique that is commonly used to minimise noise in images and signals. This noise reduction is a form of common preprocessing phase used to enhance the results of subsequent 
processing (such as edge detection on an image). Since it retains edges under certain conditions while eliminating noise, the digital image processing widely uses the median filtering technique and signal processing applications. The key concept of the median filter is to move through the input signal, replacing each input with the median of neighbouring patterns. The "port" that slides over the entire signal, entry by entry, is called the "port." The first few preceding and following entries are the most obvious window in 1D signals, while more complex window patterns (such as "box" or "loop" patterns) are possible in 2D (or higher-dimensional) signals such as images. Remember that if the window has an odd entries in number, the median is defined in a simple way: it is simply the mid value after all the entries in the window have been numerically arranged. There's more than one logical median for an even number of entries, see Median for more information.

\section{EDGE DETECTION}

Edge detection is one of technique for extracting structural information from different points of vision and for significantly reduces the amount of data to be processed. It has been implemented widely across various computer vision systems.

\subsection{CANNY EDGE DETECTION}

Canny implemented edge detection by finding the requirements on various vision systems was fairly identical. As a result, an edge detection system can be used to meet requirements in a wide range of circumstances. Edge detection with a lower rate of errors is one of general edge detection requirements, which means that detection should accurately capture as many edges as possible in the image. An operator's edge point should be correct in its position at the edge centre. An image should be marked once within provided edge, and image noise should not produce false edges where possible.

\section{IMAGE SEGMENTATION}

Image segmentation is used to separate the digital images into multiple segments (pixel sets, it is also known as image objects). The purpose of partitioning is to simplify and or make the representation of an image more meaningful and easier to analyse.

\subsection{OTSU THRESHOLDING}

In image processing and computer vision, Otsu's method for performing automated image thresholding is used. In its most simplest form, the single intensity threshold is returned from the algorithm that pixels were divided into two groups: fore-ground and background. This threshold is determined by decreasing intra-class variance, or, in other words, minimizing intra-class variance. Otsu is a one dimensional method, with Fisher's Discriminant Analysis of discrete variant, is similar to Jenk's optimization method, and is equivalent to a globally optimal k-means on the strength histogram.

\section{MORPHOLOGY}

Morphological processing of images is a set of non-linear operations related to the shape or morphology of features in an image. According to this, operations on morphology depend only on the pixel values with relative ordering, not on their numerical values, and are thus particularly suited to binary image processing. Morphological operations can be applied to grey scale images, such that light transmission functions were not known and there is no absolute pixel values or minor absolute pixel. A morphological operation on a binary image creates a new binary image in which the pixel only has a non-zero value if the experiment at that position in the input image is successful. The small binary image is an structuring element, i.e. a matrix of small pixel, each with a value of zero or one: The dimensions of the matrix determine the size of the structured element. The one and zero pattern specify the structuring element form. One of its pixels is typically an origin of the structuring element, but generally the origin can be outside the structuring element.
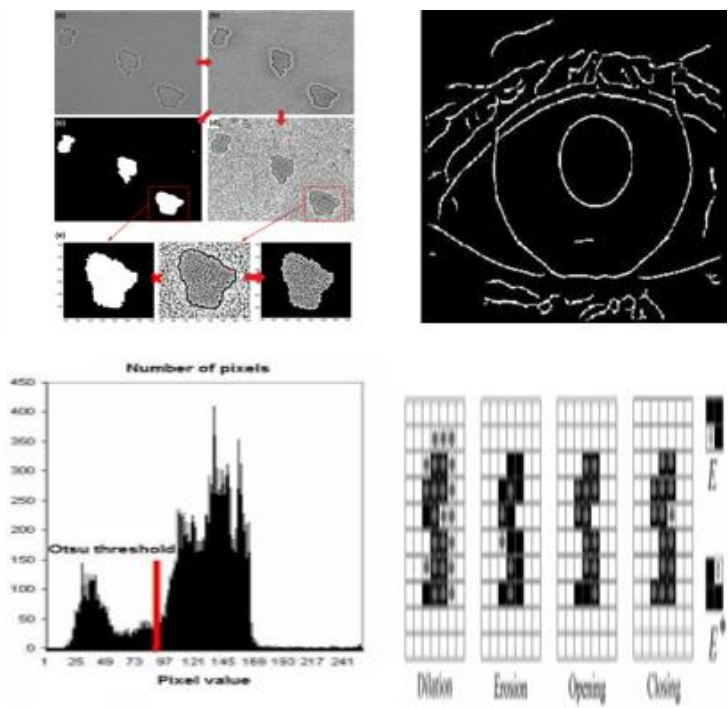

Fig. 4. Techniques of Crack Detection (a) Image Pre-processing, (b) Edge Detection, (c) Image Segmentation, and (d) Morphology.

\section{B. Data included in web server}

The data of the crack detection is given as input to the web server and locating it using the GPS location.

\section{GPS LOCATION}

The GPS (Global Positioning System) is a "constellation" of about 30 satellites orbiting the Earth that enable people 


\section{International Journal of Engineering Applied Sciences and Technology, 2021 \\ Vol. 5, Issue 10, ISSN No. 2455-2143, Pages 161-170 \\ Published Online February 2021 in IJEAST (http://www.ijeast.com)}

with terrestrial receivers to determine their geographic location. For most devices the positioning accuracy is from 10 to 100 meters everywhere. With special military approved equipment, accuracy can be defined to within one (1) meter. GPS equipment is widely used in research, and it has now become cheap enough for almost everyone to buy a GPS receiver. GPS is also one of main input to upload in web server to find exactly where the crack is located. Hence the GPS plays a vital role. The GPS location is detected using two main things are Latitude and Longitude.

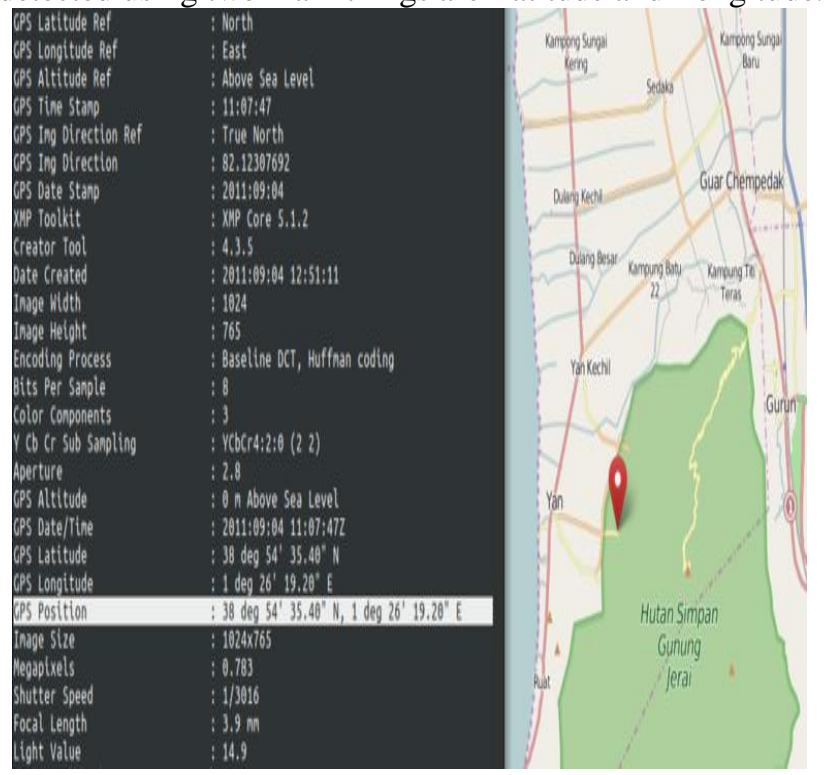

Fig. 5. Detection of GPS Location.

\subsection{LATITUDE}

The latitude shows the angle between the straight line in the particular point and the equatorial plane, and its symbol is phi. The latitude is determined by degrees, beginning at $0^{\circ}$ and ending at $90^{\circ}$ on both sides of the equator, rendering northern and southern latitude. The equator is a line with a latitude of $0^{\circ}$.

\subsection{LONGITUDE}

The longitude is another angular coordinate that determines a point's location on an earth's surface, and its symbol is lambda. The longitude is defined as an angle from the Greenwich Meridian pointing east or west which is taken as the Prime Meridian. The maximum longitude can be specified from the Prime Meridian as $180^{\circ}$ east and from the Prime Meridian as $180^{\circ}$ west.

\section{WEB SERVER UPDATE}

Using the detected crack data and finding the location using GPS, the inputs are given to web server. A web can be viewed by anyone in world. Hence updating the data into web server provides useful information and necessary department can take actions.
By this more traffic can be avoided and accidents can also be reduced. If more vehicles are passing in same road that the department can take necessary actions to overcome these problems. In future also these set of data are also very helpful. The necessary potholes and cracks can be detected, repaired and maintained.

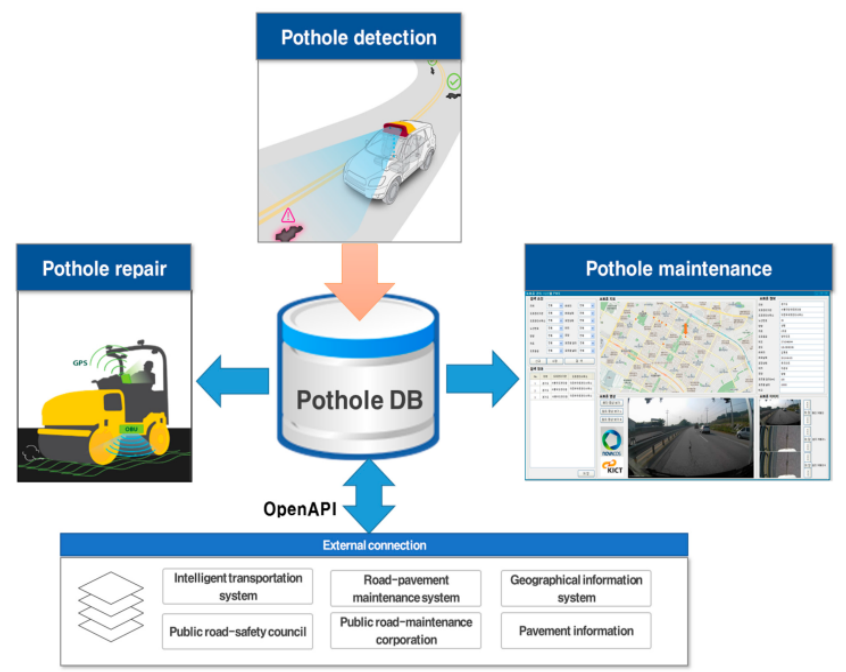

Fig. 6. Detected crack data as input and storing it in web server providing useful information.

\section{RESULT AND DISCUSSIONS}

The result of image processing deals with the image as input. The image is captured by using a camera in the vehicle. These captured images are given to the crack detection techniques, to process these images to separate the crack detected parts from the unwanted parts. The processing of image includes these processed images like original image, contrast stretched image, RGB to grey scale, segmented cracks, thinned image, filled image, cleaned image, final output image.

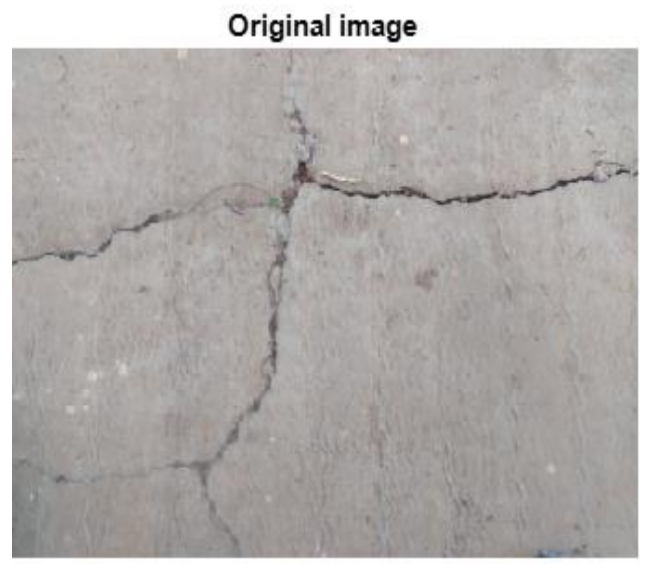

Fig. 7. Original Image of Road Crack. 
International Journal of Engineering Applied Sciences and Technology, 2021

Vol. 5, Issue 10, ISSN No. 2455-2143, Pages 161-170

Published Online February 2021 in IJEAST (http://www.ijeast.com)

\section{Contrast stretched image}

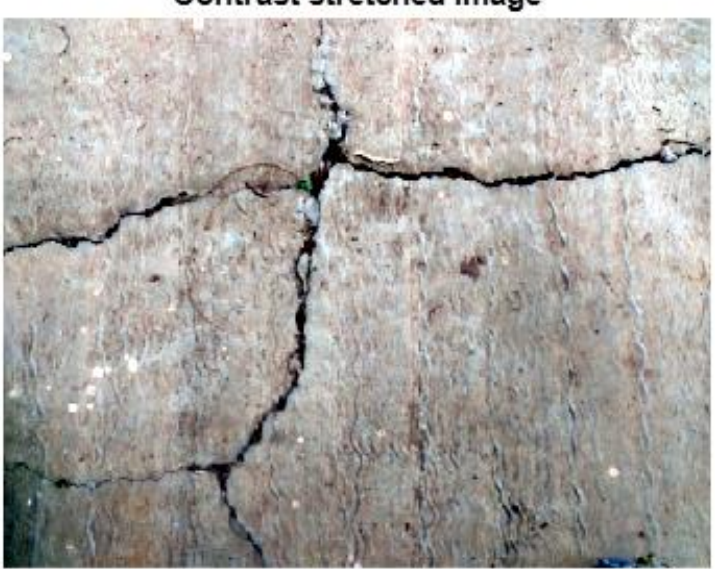

Fig. 8. Contrast Stretched Image.

\section{RGB to gray (contrast stretched)}

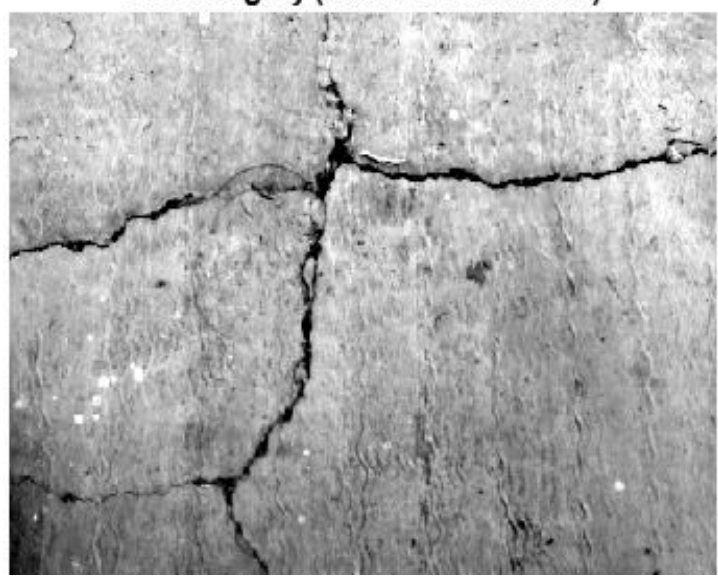

Fig. 9. RGB to Grey Scale Image.

Segmented cracks

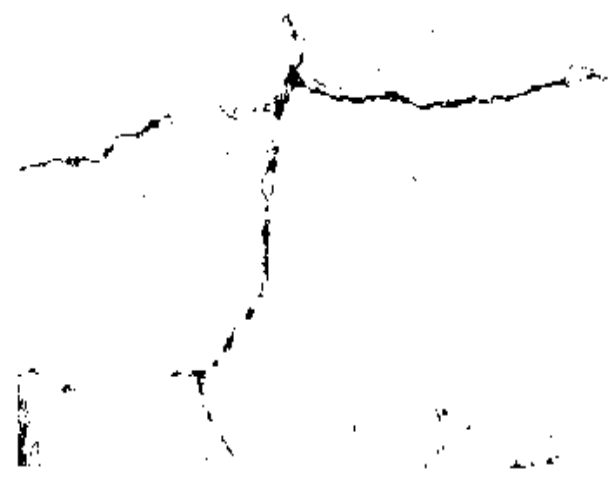

Fig. 10. Segmented Cracks.

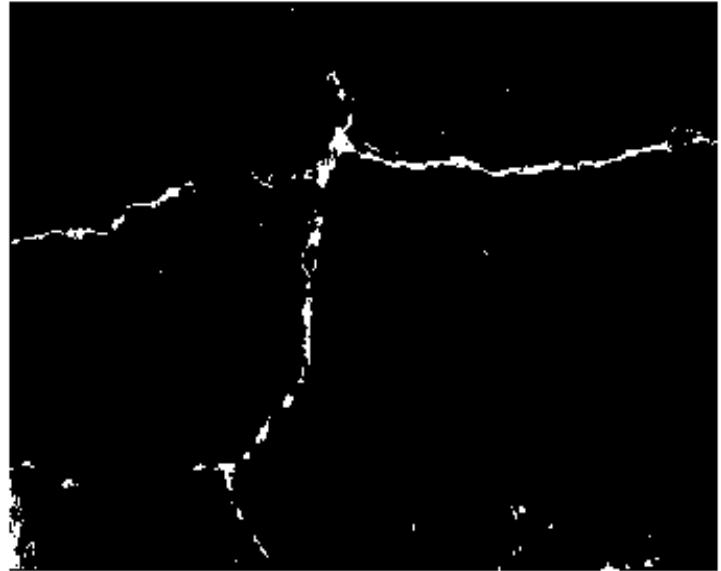

Fig. 11. Pixel Colour Changed Image.

Thinned image

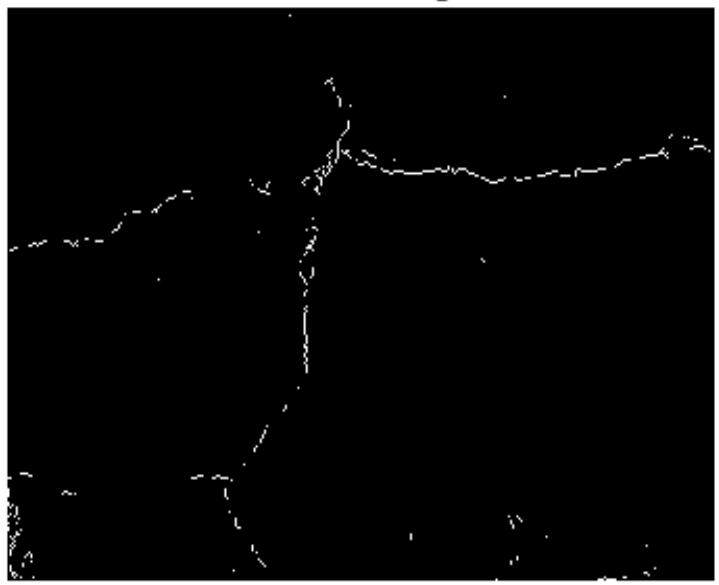

Fig. 12. Thinned Image.

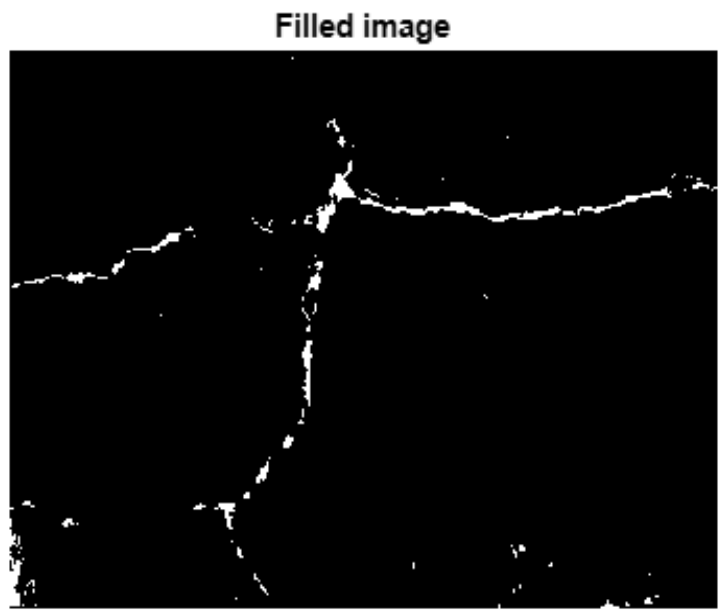

Fig. 13. Filled Image. 
International Journal of Engineering Applied Sciences and Technology, 2021

Vol. 5, Issue 10, ISSN No. 2455-2143, Pages 161-170

Published Online February 2021 in IJEAST (http://www.ijeast.com)

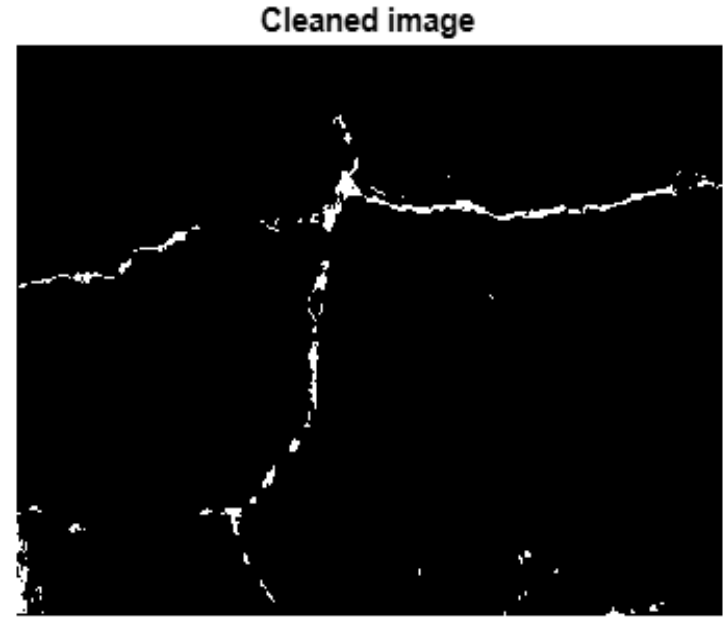

Fig. 14. Cleaned Image.

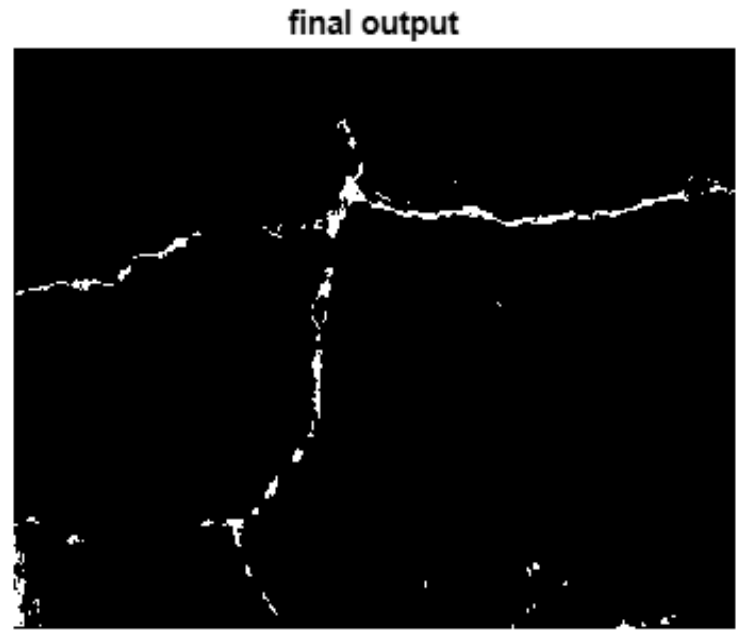

Fig.15. Final Output Image.

By these inputs, the differences between the crack and noncrack roads can be easily identified. The major difference will be there is continuous pixel lines in crack-based image, and there will be no continuous pixel lines in noncrack-based image. The output of non-cracked image as follows.

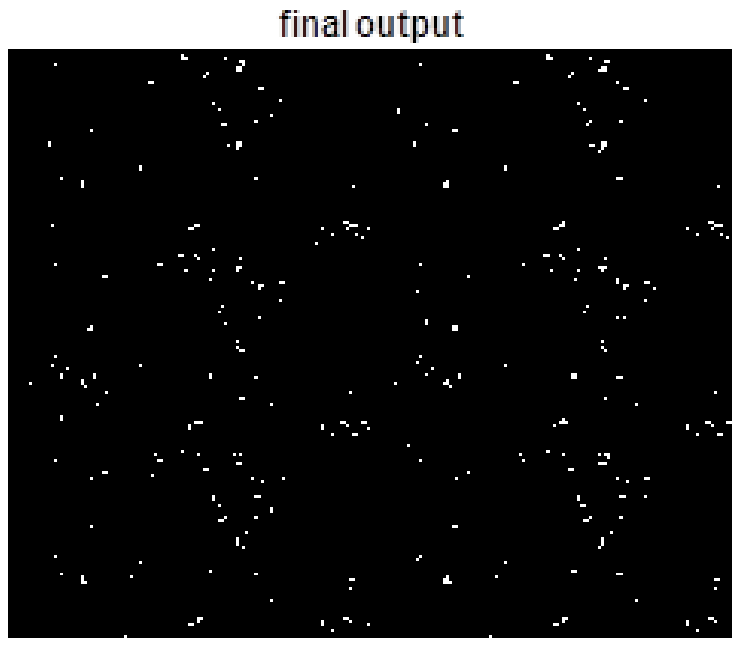

Fig. 16. An output image for non-crack-based image.

With these processed images the location is detected using GPS and these are stored in the web server.

\section{TABLE}

The following table shows the successful accuracy of pothole detection comparison

\begin{tabular}{|c|c|c|c|c|c|}
\hline Dalasct & Mcthod & $\begin{array}{c}\text { Total } \\
\text { Potholes }\end{array}$ & $\begin{array}{c}\text { Correct } \\
\text { Detection }\end{array}$ & $\begin{array}{l}\text { Lncorrect } \\
\text { Detection }\end{array}$ & Misdetection \\
\hline \multirow{3}{*}{ Dataset 1} & \multirow{3}{*}{$\begin{array}{l}\text { algorithm in }[15] \\
\text { algorithm in [16] } \\
\text { our algorithm }\end{array}$} & \multirow{3}{*}{22} & 11 & 11 & 0 \\
\hline & & & 22 & 0 & 0 \\
\hline & & & 22 & 0 & 0 \\
\hline \multirow{3}{*}{ Datasct 2} & \multirow{3}{*}{$\begin{array}{l}\text { algorithm in [15] } \\
\text { algorithm in [16] } \\
\text { our algorithm }\end{array}$} & \multirow{3}{*}{52} & 42 & 10 & 0 \\
\hline & & & 40 & 8 & 4 \\
\hline & & & 51 & 1 & () \\
\hline \multirow{3}{*}{ Dataset 3} & \multirow{3}{*}{$\begin{array}{l}\text { algorithm in [15] } \\
\text { algorithm in [16] } \\
\text { our algorithm }\end{array}$} & \multirow{3}{*}{5} & 5 & () & () \\
\hline & & & 5 & () & () \\
\hline & & & 5 & () & () \\
\hline \multirow{3}{*}{ Total } & \multirow{3}{*}{$\begin{array}{l}\text { algorithm in }|15| \\
\text { algorithm in }|16| \\
\text { our algorithm }\end{array}$} & \multirow{3}{*}{79} & 58 & 21 & () \\
\hline & & & 67 & 8 & 4 \\
\hline & & & 78 & 1 & ) \\
\hline
\end{tabular}




\section{GRAPHS}

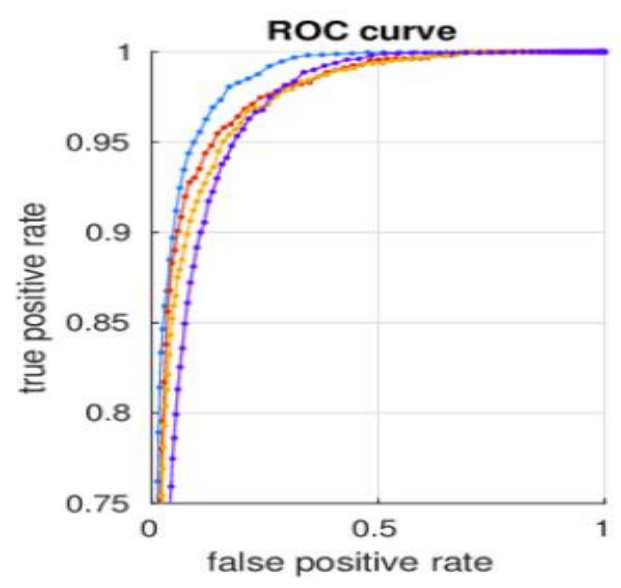

Fig.17. ROC Curve.

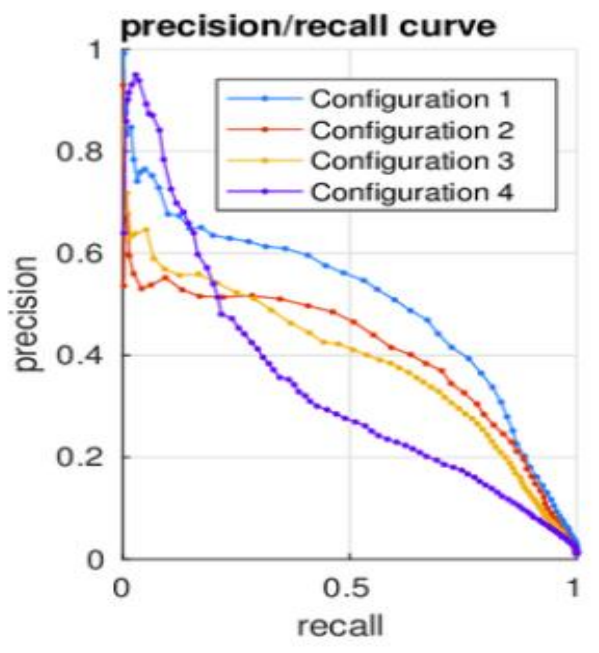

Fig.18. Precision/Recall curve.

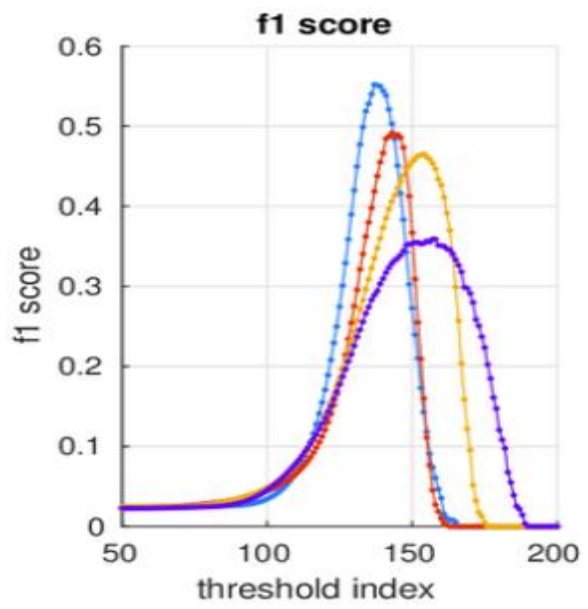

Fig.19. F1 Score.
The Graphs like ROC Curve, Precision/Recall curve, F1 Score with respect to threshold index are taken and compared with existing system with proposed system. These Graphs show the increased accuracy of our system.

\section{CONCLUSIONS AND FUTURE WORK}

This paper's key contributions are a novel method for transformation of disparity map modelling algorithm. The road area which is undamaged in the transformed disparity map can be better distinguishable with our approach, and it can be easily removed by applying the Otsu thresholding algorithm method. The robustness improved greatly of using this disparity map technique. Furthermore, in the process of map modelling of disparity, normal vectors of this disparity differs greatly from the ideal one that were discarded, and this improving accuracy of the modelled map disparity. By comparing the difference between the modelled disparity maps with the actual one, the potholes were eventually found.

The experimental results show that overall accuracy for the proposed algorithm is approximately calculated as $98.7 \%$ and the accuracy of the pixel is calculated as $99.6 \%$.

The identification of potholes with the parameters set cannot be applied to all the cases. The transformed disparity map that was planned and deep neural network is trained to identify the potholes. In addition, the quadratic is not considered for road surfaces. Thus, proposed pothole detection algorithm is implemented before, the design of algorithm that we aim to segment the reconstructed road surfaces into the group of localized planes.

\section{REFERENCES}

[1] R. Fan, X. Ai, and N. Dahnoun, "Road surface 3D reconstruction based on dense subpixel disparity map estimation," IEEE Trans. Image Process., vol. 27, no. 6, pp. 3025-3035, Jun. 2018.

[2] Wohlschlaeger M, Sauter T, Jasperneite J. The Future of Industrial Communication: Automation Networks in the Era of the Internet of Things and Industry 4.0[J]. IEEE Industrial Electronics Magazine, vol. 42, no. 11, pp. 17-27, Nov. 2017.

[3] S. Li, C. Yuan, D. Liu, and H. Cai, "Integrated processing of image and GPR data for automated pothole detection," J. Comput. Civil Eng., vol. 30, no. 6, Art. no. 04016015, Nov. 2016.

[4] Khan S M, Atamturktur S, Chowdhury M, et al. Integration of Structural Health Monitoring and Intelligent Transportation Systems for Bridge Condition Assessment: Current Status and Future Direction[J]. IEEE Transactions 


\section{International Journal of Engineering Applied Sciences and Technology, 2021 \\ Vol. 5, Issue 10, ISSN No. 2455-2143, Pages 161-170 \\ Published Online February 2021 in IJEAST (http://www.ijeast.com)}

on Intelligent Transportation Systems, vol. 395, no. 17, pp. 2107-2122, Aug. 2016.

[5] R. G. Lins S. N. Givigi "Automatic crack detection and measurement based on image analysis" IEEE Trans. Instrum. Meas. vol. 65 no. 3 pp. 583-590 Mar. 2016.

[6] F. Saeed, S. Qamariatul, M. Rahman, A. Woodside, "The state of pothole management in UK local authority" in Bituminous Mixtures and Pavements VI, Boca Raton, FL, USA:CRC Press, pp. 153-159, 2015.

[7] Farrar C R, Doebling S W, Nix D A. Vibration-based structural damage identification[J]. Philosophical Transactions of The Royal Society B Biological Sciences, vol. 395, no. 19, pp. 131-149, Sep. 2015.

[8] Hu B, Lu Z, Li H, et al. Convolutional neural network architectures for matching natural language sentences $[\mathrm{J}]$. vol. 34, no. 9, pp. 2042- 2050, Sep. 2015.

[9] R. Madli, S. Hebbar, P. Pattar, V. Golla, "Automatic Detection and Notification of Potholes and Humps on Roads to Aid Drivers", IEEE Sensors Journal, vol. 15, no. 8, pp. 4313-4318, 2015.

[10] J. S. Miller and W. Y. Bellinger, "Distress identification manual for the long-term pavement performance program," Office Infrastructure. Res. Develop., Federal Highway Admin., Washington, DC, USA, Tech. Rep. FHWA-HRT-13-092, 2014.

[11] Burgart Steven, Gap Trap: A Pothole Detection and Reporting System Utilizing Mobile Devices, 2014.

[12] T. Kim, S.-K. Ryu, "Review and analysis of pothole detection methods", J. Emerging Trends Comput. Inf. Sci., vol. 5, no. 8, pp. 603-608, 2014.

[13] T. Shen, G. Schamp, M. Haddad, "Stereo vision based road surface preview", Proc. Int. Conf. Intelligent Transportation Systems, pp. 1843-1849, October 2014.

[14] Gelenbe E. Stability of the random neural network model[J]. Neural Computation, .vol. 84, no. 12, pp. 239247, Sep. 2014.

[15] T. Kim and S. K. Ry, "Review and analysis of pothole detection methods," J. Emerg. Trends Comput. Inf. Sci., vol. 5, no. 8, pp. 603-608, Aug. 2014.

[16] Chen S, Cerda F, Rizzo P, et al. Semi-Supervised Multiresolution Classification Using Adaptive Graph Filtering With Application to Indirect Bridge Structural Health Monitoring[J]. IEEE Transactions on Signal Processing, vol.62, no. 11, pp.2879-2893, May. 2014.

[17] Z. Zhang, X. Ai, C. K. Chan, and N. Dahnoun, "An efficient algorithm for pothole detection using stereo vision," in Proc. IEEE Int. Conf. Acoust., Speech Signal Process. (ICASSP), pp. 564-568, May. 2014.
[18] Perera C, Zaslavsky A, Christen P, et al. Context Aware Computing for The Internet of Things: A Survey[J]. IEEE Communications Surveys \& Tutorials, vol. 38, no. 16, pp. 414-454, Feb. 2013.

[19] Lawrence S, Giles C L, Tsoi A C, et al. Face recognition: a convolutional neural-network approach[C] Computer Vision and Pattern Recognition, 1996. Proceedings CVPR '96, 1996 IEEE Computer Society Conference on. IEEE, vol. 74, no. 18, pp. 217-222, Sep. 2012.

[20] Q. Zou Y. Cao Q. Li Q. Mao S. Wang "CrackTree: Automatic crack detection from pavement images" Pattern Recognit. Lett. vol. 33 no. 3 pp. 227-238 2012.

[21] Atzori L, Iera A, Morabito G. SIoT: Giving a Social Structure to the Internet of Things[J]. IEEE Communications Letters, vol. 62, no. 11, pp. 1193-1195, Nov. 2011.

[22] Koch and I. Brilakis, "Pothole detection in asphalt pavement images," Adv. Eng. Inform., vol. 25, no. 3, pp. 507-515, 2011.

[23] M. Gavilán et al. "Adaptive road crack detection system by pavement classification" Sensors vol. 11 no. 10 pp. 9628-9657 2011.

[24] Y. Hu C.-X. Zhao H.-N. Wang "Automatic pavement crack detection using texture and shape descriptors" IETE Tech. Rev. vol. 27 no. 5 pp. 398-405 2010.

[25] R. Adi, M. Homji, "Intelligent Pothole Repair Vehicle", Texas A and M University, August 2005.

[26] Hasith Karunasekera, Han Wang, Handuo Zhang. "Energy Minimization Approach for Negative Obstacle Region Detection", IEEE Transactions on Vehicular Technology, 2019.

[27] Haifeng Li, Dezhen Song, Yu Liu, Binbin Li. "Automatic Pavement Crack Detection by Multi-Scale Image Fusion", IEEE Transactions on Intelligent Transportation Systems, 2019.

[28] Liyan Zhang, Guanchen Zhou, Yang Han, Honglei Lin, Yuying Wu. "Application of Internet of Things Technology and Convolutional Neural Network Model in Bridge Crack Detection", IEEEAccess, 2018.

[29] Dong-Won Jang, Rae-Hong Park. "Pothole detection using spatio-temporal saliency", IET Intelligent Transport Systems, 2016.

[30] Yiyang, Zhang. "The design of glass crack detection system based on image preprocessing technology", 2014 IEEE 7th Joint International Information Technology and Artificial IntelligenceConference, 2014. 